\title{
A New Expression of Near-Field Gain Correction Using Photonic Sensor and Planar Near-Field Measurements
}

\author{
Masanobu Hirose $\cdot$ Satoru Kurokawa
}

\begin{abstract}
We propose a new expression of the near-field gain correction to calculate the on-axis far-field gain from the onaxis near-field gain for a directive antenna. The new expression is represented by transversal vectorial transmitting characteristics of two antennas that are measured by planar near-field equipment. Due to the advantages of the photonic sensor, the utilization of the new expression realizes the measurements of the on-axis far-field gains for two kinds of double ridged waveguide horn antennas within $0.1 \mathrm{~dB}$ deviation from $1 \mathrm{GHz}$ to $6 \mathrm{GHz}$ without calibrating the photonic sensor system.
\end{abstract}

Key words: Near-Field Gain, On-Axis Gain, Photonic Sensor, Planar Near-Field Measurements.

\section{I . Introduction}

The accurate measurements of the on-axis far-field gains of antennas are of primary importance in antenna measurements. The conventional method for the on-axis far-field gains uses the transmission measurements between antennas whose distance are far enough to fulfill the far-field conditions [1], or the near-field gain corrections to the transmission measurements between antennas at finite distances less than the distances of the far-field conditions [2] [5]. As the most accurate method, the extrapolation method is used in national metrology laboratories [6].

The conventional near-field gain correction is calculated using the electric and magnetic fields at the apertures of antennas. Usually the electric and magnetic field distributions are calculated at the apertures of pyramidal horn antennas from some theoretical models [2], [4], [5]. For some complicated antennas such as real doubleridged waveguide horns, it is difficult to calculate the field distributions theoretically.

Ludwig [3] obtained the electric and magnetic fields for the near-field gain correction using spherical wave expansions determined from the far-field pattern of an antenna. This method is equivalent to the combination of the near-field gain correction and spherical near-field antenna measurements by the present point of view.

We propose a new expression of the near-field gain correction combined with the planar near-field antenna measurements [7]. From the theory of the planar near- field measurements, the transmission between antennas at any distance can be expressed by the transversal vectorial transmitting characteristics (omitting "transversal vectorial" below) of the antennas. Using the transmitting characteristics, the far-field gain can be exactly obtained. Therefore the near-field gain correction at any distance is expressed by the ratio of the far-field gain to the near-field gain in terms of the transmitting characteristics of two antennas. This is a new expression of the near-field gain correction.

Generally, it is know that the far-field gain can be obtained by the planar near-field antenna measurements if the characteristic of the probe used in the measurements is known. However, in real measurements, there are serious problems such as truncation errors and multiple reflections between the antenna and the probe [8], especially for broad beam-width antennas such as doubleridged waveguide horn antennas.

In contrast, if the photonic sensor is used as the probe, the problems are overcome because the photonic sensor is a small dielectric object [9]. However the measurement system using the photonic sensor is difficult to be calibrated in order to obtain absolute gains of antennas.

Combining the new expression and the planar nearfield measurement using the photonic sensor, we can obtain the on-axis far-field gain without calibrating the photonic sensor system.

In the following sections, the near-field gain correction is defined and expressed in a new form. To validate

Manuscript received August 25, 2011 ; Revised February 14, 2012 ; Accepted March 5, 2012. (ID No. 20110825-07J)

National Metrology Institute of Japan, National Institute of Advanced Industrial Science and Technology, Ibaraki, Japan.

Corresponding Author : Masanobu Hirose (e-mail : masa-hirose@aist.go.jp)

This is an Open-Access article distributed under the terms of the Creative Commons Attribution Non-Commercial License (http://creativecommons.org/licenses/ by-nc/3.0) which permits unrestricted non-commercial use, distribution, and reproduction in any medium, provided the original work is properly cited. 
the new expression, some simulation results are shown. Finally the measurement results of double-ridged waveguide horn antennas using the photonic sensor demonstrate the usefulness of the new expression.

\section{II . Near-Field Gain Correction}

\section{2-1 Conventional Expression}

To obtain the on-axis far-field gain of an antenna that is defined on the far-field condition, the correction to the on-axis near-field gain of the antenna is required. The near-field gain correction (NGC) is defined by the ratio of the far-field gain $G$ to the near-field gain $G_{\mathrm{NF}}$ as

$$
N G C=\frac{G}{G_{N F}} .
$$

Assuming that two antennas designated as 1 and 2 are separated at a distance $z$, the product of the near-field gains for each antenna is defined as

$$
G_{N F 1}(z) G_{N F 2}(z)=\frac{1}{M_{1} M_{2}}\left(\frac{4 \pi z}{\lambda}\right)^{2} \frac{P_{r e c 1}}{P_{\text {in } 2}}
$$

using the Friis transmission formula [10] where $M_{i}(i=1$, 2 ) is the mismatch power loss of antenna $i, \lambda$ is wavelength, $P_{\text {rec } 1}$ and $P_{i n 2}$ are the received power by antenna 1 and the input power (not the transmitted power) into antenna 2 respectively. Throughout the paper, we assume that the antennas are reciprocal and multiple reflections between the antennas can be neglected using a filtering method of the transmission data [3]. The farfield gain $G_{i}$ of the antenna $i$ is related to the near-field gain as

$$
G_{i}=\lim _{z \rightarrow+\infty} G_{N F i}(z) .
$$

The power transmission formula is given as [2] [5]

$$
\frac{P_{r e c}}{P_{i n}}=\frac{\left|\int_{S}\left(\mathbf{H}_{2} \times \mathbf{E}_{1}+\mathbf{E}_{2} \times \mathbf{H}_{1}\right) \cdot \hat{\mathbf{n}} d s\right|^{2}}{4\left\{\operatorname{Re} \int_{S_{1}} \mathbf{E}_{1} \times \mathbf{H}_{1}^{*} \cdot \hat{\mathbf{n}}_{1} d s_{1}\right\}\left\{\operatorname{Re} \int_{S_{2}} \mathbf{E}_{2} \times \mathbf{H}_{2}^{*} \cdot \hat{\mathbf{n}}_{2} d s_{2}\right\}}
$$

where $\mathbf{E}_{1}$ and $\mathbf{H}_{1}$ are the fields when antenna 1 is transmitting, $\mathbf{E}_{2}$ and $\mathbf{H}_{2}$ are the fields when antenna 2 is transmitting, $S_{1}$ and $S_{2}$ are surfaces enclosing antennas 1 and 2 respectively, $S$ is a surface enclosing either anten$\mathrm{na}, \widehat{\mathbf{n}}, \widehat{\mathbf{n}}_{1}$, and $\widehat{\mathbf{n}}_{\mathbf{2}}$ are the unit normals to the surfaces, and $*$ means taking the complex conjugate.

To calculate (4), we must obtain the fields $\mathbf{E}_{\mathrm{i}}$ and $\mathbf{H}_{\mathrm{i}}$ radiated by antenna $i$ on each surface. They are calculated by the assumed field distributions at the antenna apertures [2], [4], [5] or obtained by the spherical wave expansions whose coefficients are determined by the far-field pattern of the antenna $i$ [3].

We define a ratio

$$
C_{12}(z)=\frac{\lim _{z \rightarrow+\infty} z|| \int_{S}\left(\mathbf{H}^{\prime}{ }_{2} \times \mathbf{E}^{\prime}{ }_{1}+\mathbf{E}^{\prime}{ }_{2} \times \mathbf{H}_{1}{ }_{1}\right) \cdot \hat{\mathbf{n}} d s \mid}{z\left|\int_{S}\left(\mathbf{H}_{2} \times \mathbf{E}_{1}+\mathbf{E}_{2} \times \mathbf{H}_{1}\right) \cdot \hat{\mathbf{n}} d s\right|}
$$

where quantities with primes correspond the ones in the case where the antenna distance is $z^{\prime}$. Using (2) to (5), we can get

$$
C_{12}(z)=\sqrt{\frac{\left(G_{1} G_{2}\right)}{\left\{G_{N F 1}(z) G_{N F 2}(z)\right\}}}
$$

because $M_{1}, M_{2}$, and the denominator of (4) are independent on $z$. Combining the three antenna method [1], we finally obtain the NGC for antenna 1 as

$$
\begin{aligned}
N G C_{1}(z)= & \sqrt{\frac{\left(G_{1} G_{2}\right)}{\left\{G_{N F F}(z) G_{N F 2}(z)\right\}} \frac{\left(G_{1} G_{3}\right)}{\left\{G_{N F 1}(z) G_{N F 3}(z)\right\}} \frac{\left\{G_{N F 2}(z) G_{N F 3}(z)\right\}}{\left(G_{2} G_{3}\right)}} \\
& =\frac{C_{12}(z) C_{13}(z)}{C_{23}(z)}
\end{aligned}
$$

where subscript 3 represents quantities for antenna 3. If antenna 1 and antenna 2 have completely the same characteristics, (7) reduces to (1) as

$$
N G C_{1}(z)=\sqrt{\frac{\left(G_{1} G_{1}\right)}{\left\{G_{N F 1}(z) G_{N F 1}(z)\right\}}}=C_{11}(z) .
$$

This means that $C_{i j}(z)(i, j=1,2,3)$ can be considered as an extended NGC.

Therefore the NGC is expressed by the electric and magnetic fields radiated by each antenna in the measurements.

\section{2-2 New Expression}

A new expression is derived from quantities closely related to antenna far-field patterns, that is, the transmission characteristics of antennas obtained by the planar near-field antenna measurements [7].

The extended NGC between antenna 1 and 2 is expressed as [11]

$$
C_{12}(z)=\frac{\lim _{z^{\prime} \rightarrow+\infty}\left|z^{\prime} S_{12}\left(z^{\prime}\right) e^{j k z^{\prime}}\right|}{\left|z S_{12}(z) e^{j k z}\right|}
$$

using (5) and the power transmission formula given as

$$
\frac{P_{r e c}}{P_{i n}}=\left|S_{12}(z)\right|^{2}
$$

where $S_{12}(z)$ is the $S$ parameter between antennas 1 and 2. The phase factors $e^{j k z}$ in (9) is included for the numerical integration of the denominator to be done easily 
explained later.

Owing to [7], the $S$ parameter between antennas 1 and 2 is given as

$$
S_{12}(z)=\int_{-\infty}^{+\infty} \mathbf{S}_{01}(\mathbf{K}) \cdot \mathbf{S}_{20}(\mathbf{K}) e^{-j \gamma z} d \mathbf{K}
$$

where $\mathbf{S}_{01}$ is the transversal vectorial receiving characteristic of the antenna $1, \mathbf{S}_{20}$ is the transmitting characteristic of the antenna $2, \mathbf{R}$ and $z$ are the transversal vector, the $z$ component of the position vector of the antenna 2 relative to the antenna 1 respectively, $\mathbf{K}$ and $\gamma$ are the transversal vector and the $z$ component of the wavenumber vector $\boldsymbol{k}$ respectively. $\mathbf{S}_{i 0}$ is related to the transversal electric field $\mathbf{E}_{i t}\left(\mathbf{R}, z_{i}\right)$ at the position $\left(\mathbf{R}, z_{i}\right)$, radiated by antenna $i$ as

$$
\mathbf{S}_{i 0}(\mathbf{K})=\frac{e^{j \gamma z_{i}}}{2 \pi a_{0}} \int \mathbf{E}_{i t}\left(\mathbf{R}, z_{i}\right) e^{+j \mathbf{K} \cdot \mathbf{R}} d \mathbf{K}
$$

where $a_{0}$ is the incoming wave amplitude to antenna $i$.

Inserting (11) into (9), (9) reduces to

$$
\begin{aligned}
C_{12}(z) & =\frac{\lim _{z^{\prime} \rightarrow+\infty}\left|z^{\prime} \int \mathbf{S}_{01}(\mathbf{K}) \cdot \mathbf{S}_{20}(\mathbf{K}) e^{j z^{\prime}(k-\gamma)} d \mathbf{K}\right|}{\left|z \int \mathbf{S}_{01}(\mathbf{K}) \cdot \mathbf{S}_{20}(\mathbf{K}) e^{j z(k-\gamma)} d \mathbf{K}\right|} \\
& =\frac{\left|2 \pi k \mathbf{S}_{01}(\mathbf{0}) \cdot \mathbf{S}_{20}(\mathbf{0})\right|}{\left|z \int \mathbf{S}_{01}(\mathbf{K}) \cdot \mathbf{S}_{20}(\mathbf{K}) e^{j z(k-\gamma)} d \mathbf{K}\right|} .
\end{aligned}
$$

The integral of the numerator is evaluated by the stationary phase method [10].

Since antennas are assumed to be reciprocal and used in the transmitting mode for the planar near-field measurements, it is convenient to express (13) in only the transmitting characteristics. From the reciprocity [7], $\mathbf{S}_{10}(\mathbf{K})$ is related to $\mathbf{S}_{01}(\mathbf{K})$ as

$$
\begin{aligned}
& \eta_{0} \mathbf{S}_{01}(\mathbf{K}) \cdot \hat{\mathbf{\kappa}}_{1}=\frac{\omega \varepsilon}{\gamma} \mathbf{S}_{10}(-\mathbf{K}) \cdot \hat{\mathbf{\kappa}}_{1} \\
& \eta_{0} \mathbf{S}_{01}(\mathbf{K}) \cdot \hat{\mathbf{\kappa}}_{2}=\frac{\gamma}{\omega \mu} \mathbf{S}_{10}(-\mathbf{K}) \cdot \hat{\mathbf{\kappa}}_{2}
\end{aligned}
$$

where $\eta_{0}$ is the characteristic admittance of the measurement system and $\hat{\mathbf{\kappa}}_{1}=\mathbf{K} /|\mathbf{K}|, \mathbf{\kappa}_{2}=\hat{\mathbf{z}} \times \hat{\mathbf{\kappa}}_{1}$.

Inserting (14) into (13), we obtain the new expression as

$$
C_{12}(z)=\frac{\left|2 \pi k \mathbf{S}_{10}(\mathbf{0}) \cdot \mathbf{S}_{20}(\mathbf{0})\right|}{\left|z \int \frac{1}{k \gamma}\left[\gamma^{2} \mathbf{S}_{10}(-\mathbf{K}) \cdot \mathbf{S}_{20}(\mathbf{K})+\left\{\mathbf{K} \cdot \mathbf{S}_{10}(-\mathbf{K})\right\}\left\{\mathbf{K} \cdot \mathbf{S}_{20}(\mathbf{K})\right\}\right] e^{j z(k-\gamma)} d \mathbf{K}\right|} .
$$

This expression has only transmitting characteristics that are closely related to the far-field pattern [7] and explicitly shows the dependence on the separation $z$ between antennas. We clearly find how the denominator approaches to the numerator as $z$ increases to infinity, that is, how the on-axis near-field gain converges to the on-axis far-field gain.

In practical point of view, it is difficult to perform the two-dimensional numerical integration of the denominator in (15) because the phase variation of the phase term $\exp \{j z(k-\gamma)\}$ becomes rapid when $|\boldsymbol{K}|$ approaches $k$ in the case where $z$ is a few meters even at $1 \mathrm{GHz}$. However the phase variation of $\mathbf{S}_{10}(-\mathbf{K}) \cdot \mathbf{S}_{20}(\mathbf{K})$ is slow. Therefore it is convenient to transform the integral shown below.

Since the phase term is only depend on the magnitude of $\mathbf{K}\left(k K_{n}\right.$ where $K_{n}$ is $K$ normalized by $\left.k\right)$, we first integrate along the angle $\phi$ of $\mathbf{K}$ and define a function as

$$
F\left(K_{n}\right)=\int_{0}^{2 \pi} \frac{1}{k}\left[\gamma^{2} \mathbf{S}_{10}(-\mathbf{K}) \cdot \mathbf{S}_{20}(\mathbf{K})+\left\{\mathbf{K} \cdot \mathbf{S}_{10}(-\mathbf{K})\right\}\left\{\mathbf{K} \cdot \mathbf{S}_{20}(\mathbf{K})\right\}\right] d \varphi .
$$

Next integrating along $K_{n}$ and changing it to two variables depending on the integration intervals, we have the form suitable to the numerical integration of the denominator as

$$
\begin{aligned}
\mid z \int \frac{1}{k \gamma} & {\left[\gamma^{2} \mathbf{S}_{10}(-\mathbf{K}) \cdot \mathbf{S}_{20}(\mathbf{K})+\left\{\mathbf{K} \cdot \mathbf{S}_{10}(-\mathbf{K})\right\}\left\{\mathbf{K} \cdot \mathbf{S}_{20}(\mathbf{K})\right\}\right] e^{j z(k-\gamma)} d \mathbf{K} \mid } \\
& \left.=\mid k z\left\{\int_{0}^{1} F(\sqrt{\tau(2-\tau)}) e^{j k \tau} d \tau+j \int_{0}^{+\infty} F\left(\sqrt{1+\varepsilon^{2}}\right)\right) e^{j k z-k \varepsilon} d \varepsilon\right\}
\end{aligned}
$$

where

$$
\begin{array}{ll}
\tau=1-\sqrt{1-K_{n}^{2}} & 0 \leq K_{n} \leq 1 \\
\varepsilon=\sqrt{K_{n}^{2}-1} & 1 \leq K_{n} \leq \infty .
\end{array}
$$

The first and second terms in (17) correspond to the propagation and evanescent modes [7]. Since the phase variation of the phase term in the first term is constant and the second term attenuates exponentially, it is easy to integrate (17) numerically.

\section{Numerical Simulation}

To validate the new expression, a numerical simulation using R-band standard pyramidal horn antennas is performed as shown in Fig. 1.

The antenna models the R-band standard pyramidal horn antenna (MI 12-1.7 produced by MI Technologies) whose aperture has dimensions of $272 \mathrm{~mm}$ ( $2 a$, E plane) $\times 367.5 \mathrm{~mm}(2 b, \mathrm{H}$ plane $) \times 271 \mathrm{~mm}(l$, Height $)$. All symbols for dimensions follow [5]. The waveguide part has a cross section of dimensions $109.2 \mathrm{~mm}\left(2 s_{1}\right) \times 54.6 \mathrm{~mm}$ $\left(2 s_{2}\right)$ and a length of $150 \mathrm{~mm}$. The E plane, the $\mathrm{H}$ plane, and the on-axis line of the antennas are coincident to the $y=0$ plane, the $x=0$ plane, the $z$ axis respectively. The waveguides are excited and received by only $\mathrm{TE}_{10}$ mode. 


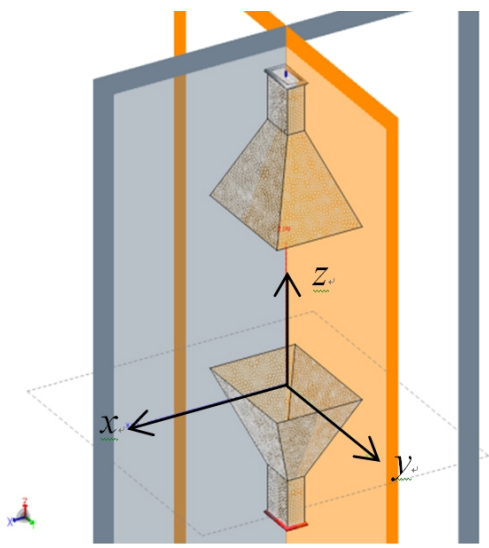

Fig. 1. Simulation model of R-band standard pyramidal horn antennas at the distance of $0.5 \mathrm{~m}$.

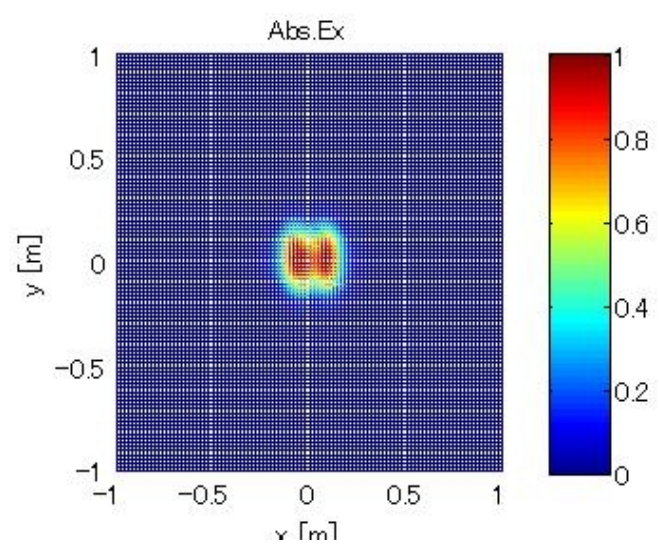

(a) $x$ component of the electric field

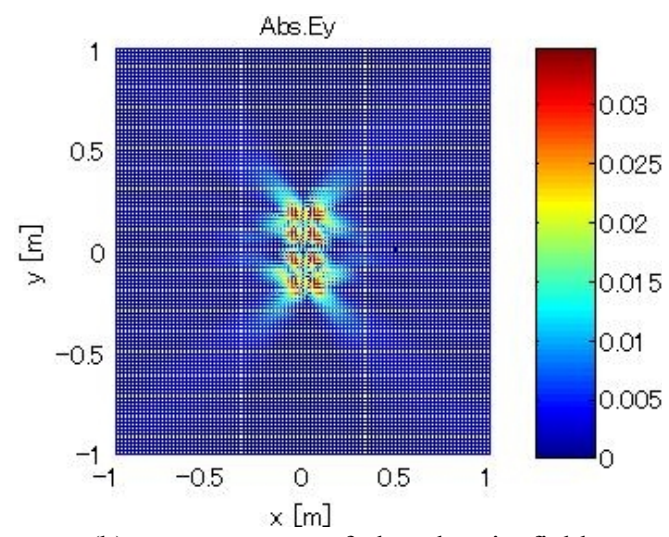

(b) $y$ component of the electric field

Fig. 2. Electric field distribution at $z=60 \mathrm{~mm}$ plane of dimensions $2 \mathrm{~m} \times 2 \mathrm{~m}$ and $2.45 \mathrm{GHz}$ in linear scale.

The simulation was done using FEKO [12] that uses the method of moments. The calculated frequency is $2.45 \mathrm{GHz}$. The edge size of all parts except excitation ports is $10 \mathrm{~mm}$ and the excitation ports are meshed in $5 \mathrm{~mm}$. The electric and magnetic symmetries are used for the $\mathrm{H}$ plane and the $\mathrm{E}$ plane respectively.

\section{3-1 Transmitting Characteristics of Antenna}

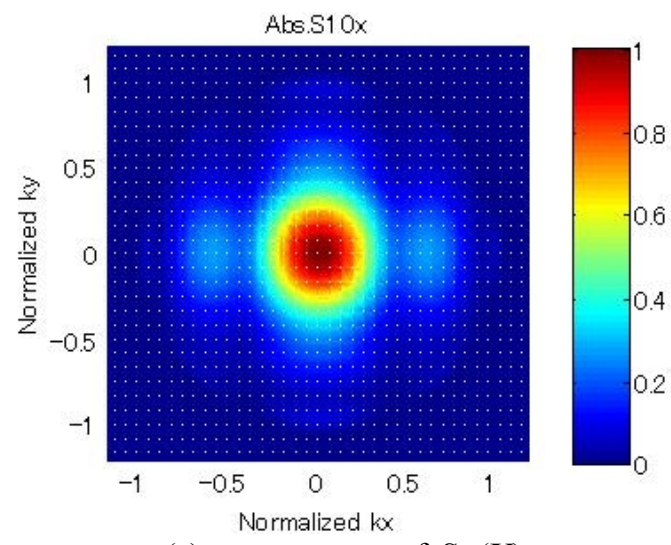

(a) $x$ component of $\mathbf{S}_{10}(\mathbf{K})$

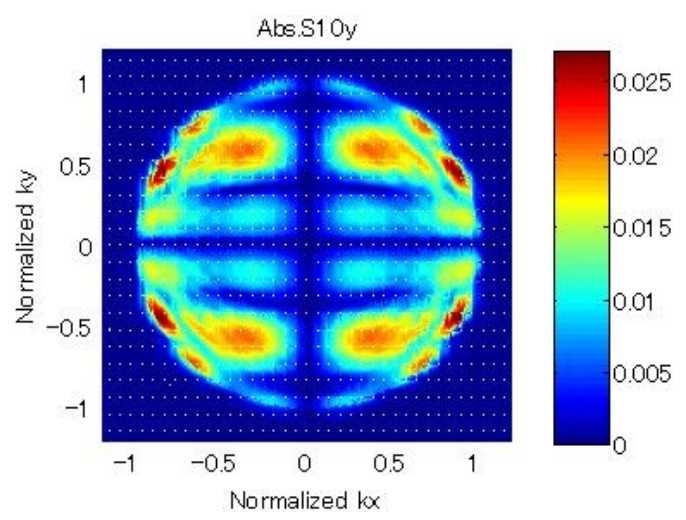

(b) $y$ component of $\mathbf{S}_{10}(\mathbf{K})$

Fig. 3. Transmitting characteristic $\mathbf{S}_{10}(\mathbf{K})$ in $\mathbf{K}$ space whose magnitude is normalized by $k$ at $2.45 \mathrm{GHz}$ in linear scale.

The transmitting characteristic $\mathbf{S}_{10}(\mathbf{K})$ of the antenna is calculated using the electric near-field data on the plane of dimensions $2 \mathrm{~m} \times 2 \mathrm{~m}$ at $z=60 \mathrm{~mm}$ with $20 \mathrm{~mm}$ steps by the planar near-field antenna method [7]. Fig. 2 shows the $x$ and $y$ components of the electric nearfield.

Fig. 3 shows the $x$ and $y$ components of $\mathbf{S}_{10}(\mathbf{K})$ that is the two-dimensional Fourier transform of the electric field.

In Fig. 2, the near-field distribution is concentrated around the antenna aperture and the $y$ component is negligibly small. Therefore the $x$ component of $\mathbf{S}_{10}(\mathbf{K})$ has no ripples due to the truncation error [8] and the $y$ component is negligible in Fig. 3. Indeed there is no difference between $\mathbf{S}_{10}(\mathbf{K})$ calculated by the near-field data on the two planes: $2 \mathrm{~m} \times 2 \mathrm{~m}$ and $4 \mathrm{~m} \times 4 \mathrm{~m}$. The far-field pattern calculated by FEKO agreed with that of $\mathbf{S}_{10}(\mathbf{K})$ within the magnitude of 0.005 .

\section{3-2 Near-Field Gain Correction}

The magnitude and phase of $\mathbf{S}_{10}(-\mathbf{K}) \cdot \mathbf{S}_{10}(\mathbf{K})$ are shown in Fig. 4. As expected, the amplitude is more 


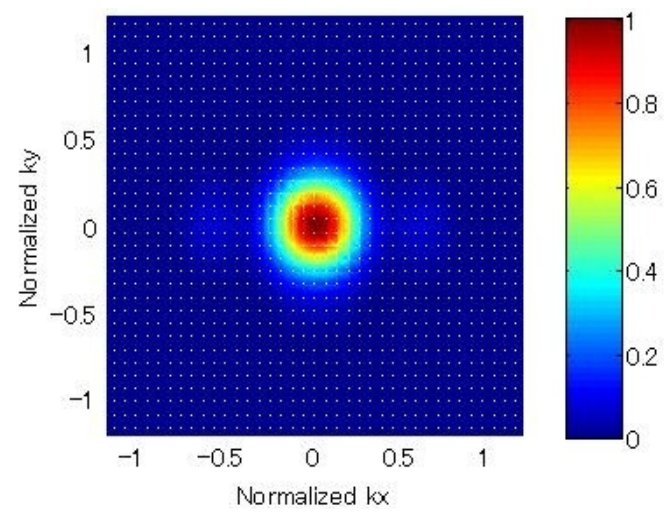

(a) Magnitude of $\mathbf{S}_{10}(-\mathbf{K}) \cdot \mathbf{S}_{10}(\mathbf{K})$

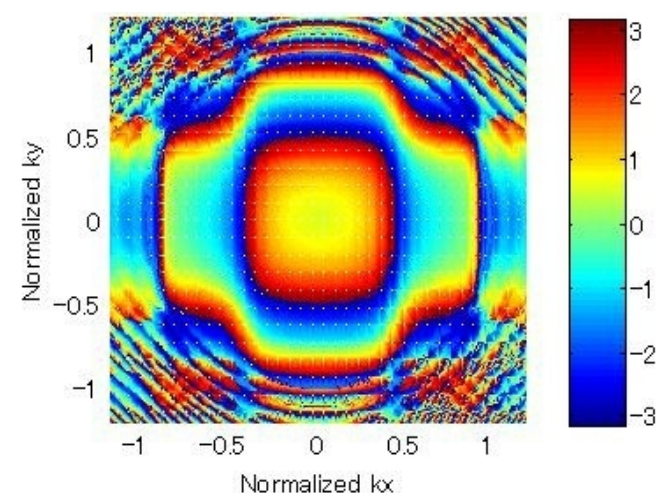

(b) Phase of $\mathbf{S}_{10}(-\mathbf{K}) \cdot \mathbf{S}_{10}(\mathbf{K})$

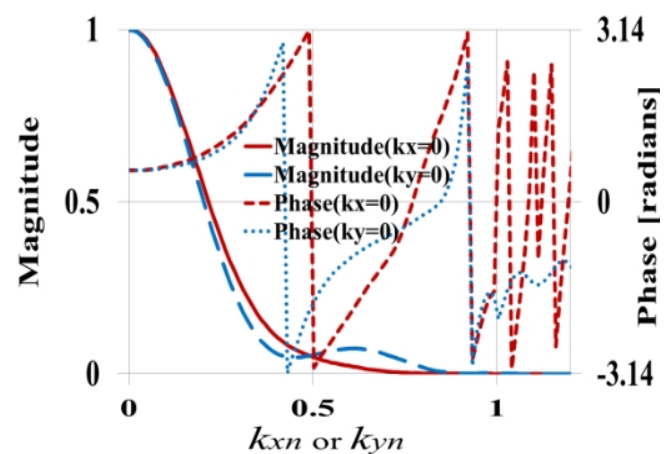

(c) Magnitude and phase of $\mathbf{S}_{10}(-\mathbf{K}) \cdot \mathbf{S}_{10}(\mathbf{K})$ at the $k_{x}=0$ and $k_{y}=0$ cut-planes

Fig. 4. Distribution of $\mathbf{S}_{10}(-\mathbf{K}) \cdot \mathbf{S}_{10}(\mathbf{K})$ in $\mathbf{K}$ space normalized to $k$.

concentrated around the origin than that of $\mathbf{S}_{10}(\mathbf{K})$ and the phase variation is slow as in Fig. 4. Therefore the phase variation of the integrand in (16) is slow and the numerical integration of (16) can be done as follows:

1) The sampling intervals of $K_{n}$ and $\phi$ are taken to be 0.02 and 5 degrees,

2) Interpolation at the sampling points in $\left(K_{n}, \phi\right)$ from the original square grid points in $\mathbf{K}$ space is done using two dimension splines,

3) And the summation around $\phi$ at the constant $K_{n}$ is taken as the integration by $\phi$.

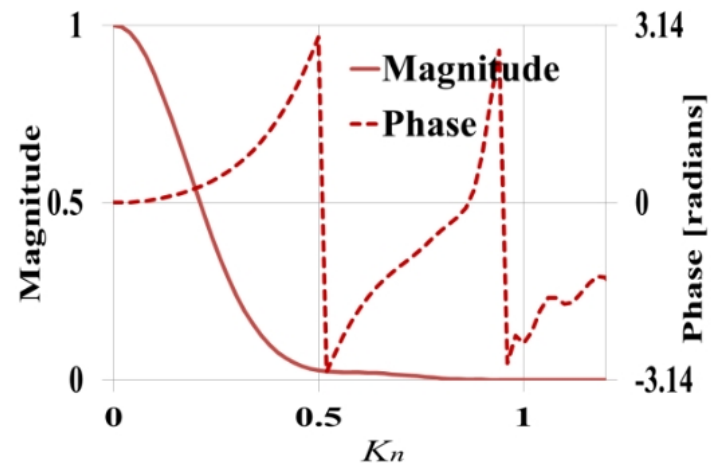

Fig. 5. Magnitude and phase of $\mathrm{F}\left(K_{n}\right)$ normalized to the value at $K_{n}=0$.

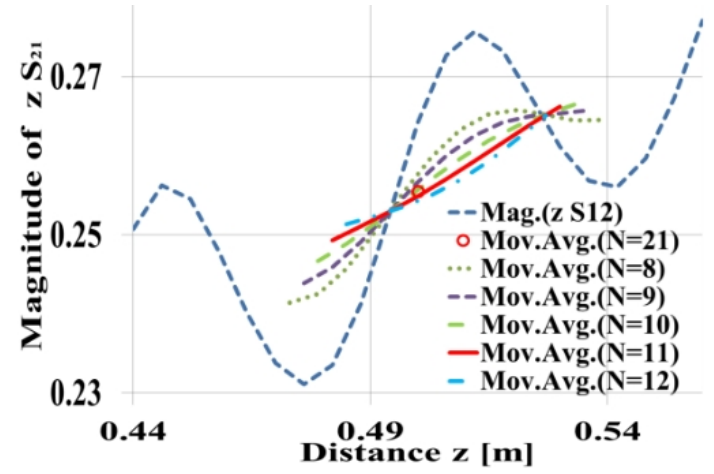

Fig. 6. Magnitude of $z S_{21}(z)$ and the moving average values in the interval of $\mathrm{N}$ points.

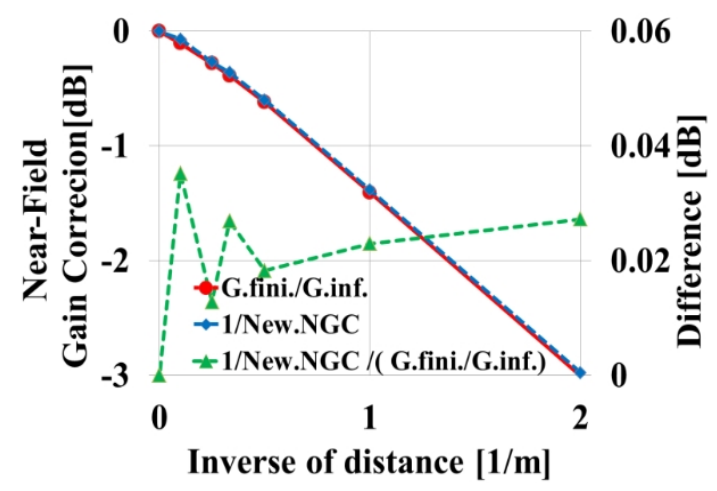

Fig. 7. Comparison of the NGC in (1) and the new NGC in (15) using the averaged values $(N=21)$ of $z S_{21}$ (z) by FEKO.

Following the above steps, we obtained the magnitude and phase of $\mathrm{F}\left(K_{n}\right)$ as shown in Fig. 5. The variation of the magnitude and the phase are similar to the ones in Fig. 4(c) as expected.

To avoid the multiple reflection in $S_{21}(z)$ by FEKO, the moving average of $z S_{21}(z) \exp (+j k z)$ is done in the interval of $120 \mathrm{~mm}$ (21 points) around the center. An example demonstrating the effect for $z=0.5 \mathrm{~m}$ (worst case) is shown in Fig. 6 and shows that the effect of the 
multiple reflection is reduced within $0.02 \mathrm{~dB}$ : The main component is the term including $\exp (-j 2 k z)$ and $N=11$ (the interval of $60 \mathrm{~mm}$ ) is the best for cancelling it $(0.5$ $\lambda=61.2 \mathrm{~mm}$ ) among $N=8$ to 12 . However we adopted $N=21$ since it is a simple average using all points.

Using $\mathrm{F}\left(K_{n}\right)$ and (17), we can calculate the NGC at any antenna separation. To validate the NGC by (15) or (16) and (17), we calculated the near-field gains in (2) using the averaged values $(N=21)$ of $z S_{21}(z)$ simulated by FEKO at the five separations $(z=0.5 \mathrm{~m}, 1 \mathrm{~m}, 2 \mathrm{~m}$, $3 \mathrm{~m}, 4 \mathrm{~m}$, and $10 \mathrm{~m}$ ) and the far-field gain by FEKO. Then we compare the NGC in (1) by FEKO with the new NGC in (15) as shown in Fig. 7.

In Fig. 7, the NGC calculated by (1) and the one by (15) agrees within $0.04 \mathrm{~dB}$ whose difference comes from mainly the interpolation error in (16). Therefore the new NGC in (15) is valid.

\section{Measurement}

To demonstrate the effectiveness of the new NGC, we have applied it to determine the gains of two kinds of double-ridged waveguide horn antennas from $1 \mathrm{GHz}$ to $6 \mathrm{GHz}$ with $0.5 \mathrm{GHz}$ steps. The antennas are often used in Electromagnetic Compatibility (EMC) measurements and have the same product name (ETS-Lindgren 3115), whereas the structures and the antenna characteristics are different. In the following, we distinguish between those by specifying the old one (discontinued) and the new one (available at present).

\section{4-1 Measurement Steup}

We used the planar near-field antenna equipment shown in Fig. 8 and a photonic sensor shown in Fig. 9.

The gain of the amplifier (HP8348A) is about $35 \mathrm{~dB}$. The output power and the IF of the vector network analyzer (VNA, Agilent E8363C) were $0 \mathrm{dBm}$ and $100 \mathrm{~Hz}$ respectively. The scanned range was $1.8 \mathrm{~m} \times 1.8 \mathrm{~m}$ with

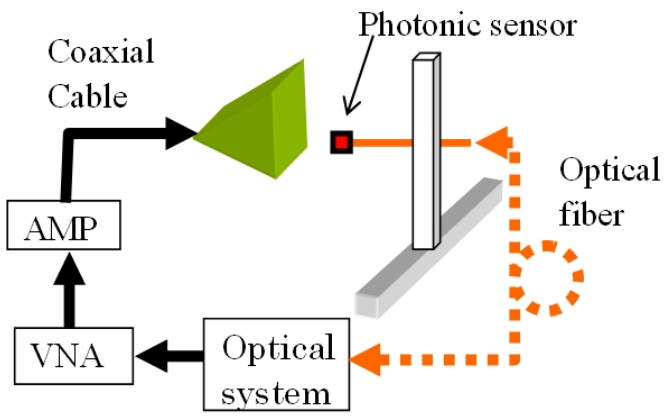

Fig. 8. Planar near-field measurement system using the photonic sensor as the probe.

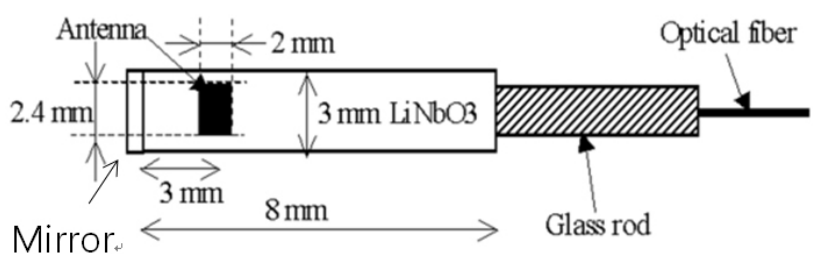

(a) Geometry

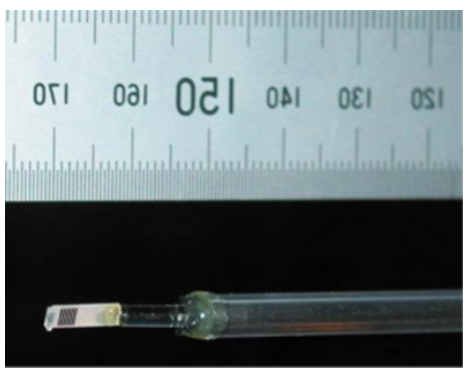

(b) Picture

Fig. 9. Photonic sensor as the probe.

$10 \mathrm{~mm}$ steps in the planar scanner (NSI $300 \mathrm{~V}-8 \times 8$ ). The distance between the aperture of each antenna and the photonic sensor was $58 \mathrm{~mm}$. The principle of the operation of the photonic sensor is explained minutely in [9].

\section{4-2 Results of Planer Near-Field Measurements}

Because the photonic sensor can measure the transversal electric field (except proportional constant) radiated by an antenna, the new NGC can be obtained in the same procedure as in the simulation by FEKO.

$S_{21 i}(\mathbf{R}, z), S$-parameter measured by the VNA, is proportional to the electric field $\mathbf{E}_{i}(\mathbf{R}, z)$ transmitted by the antenna $i$ at the sensor position $(\mathbf{R}, z)$ as

$$
S_{21 i}(\mathbf{R}, z)=\alpha_{i} \hat{\mathbf{p}} \cdot \mathbf{E}_{i}(\mathbf{R}, z)
$$

where $\alpha_{i}$ is a constant including the error terms of the measurement system and the reflection coefficient of the antenna $i, \hat{\mathbf{p}}$ is the unit vector parallel to the dipole moment vector of the photonic sensor [9], [11].

As typical characteristics, the data at $3.5 \mathrm{GHz}$ (the center frequency in the measurements) are shown below.

Fig. 10 shows the $x$ components of $\mathbf{S}_{i 0}(\mathbf{K})$ of the old type ( $i=1$, antenna 1$)$ and the new type $(i=2$, antenna 2$)$. Since the antennas are linearly polarized in the $x$ direction, the $y$ components are negligible and not shown here.

Fig. 11 shows the magnitude and the phase of $\mathbf{S}_{10}$ $(-\mathbf{K}) \cdot \mathbf{S}_{20}(\mathbf{K})$. The ones of $\mathbf{S}_{20}(-\mathbf{K}) \cdot \mathbf{S}_{20}(\mathbf{K})$ are almost the same as those. $\mathrm{F}\left(K_{n}\right)$ for $\mathbf{S}_{10}(-\mathbf{K}) \cdot \mathbf{S}_{20}(\mathbf{K})$ and $\mathbf{S}_{20}$ $(-\mathbf{K}) \cdot \mathbf{S}_{20}(\mathbf{K})$ are depicted in Fig. 12.

As seen in Fig. $10, \mathbf{S}_{i 0}(\mathbf{K})(i=1,2)$ at $3.5 \mathrm{GHz}$ are dif- 


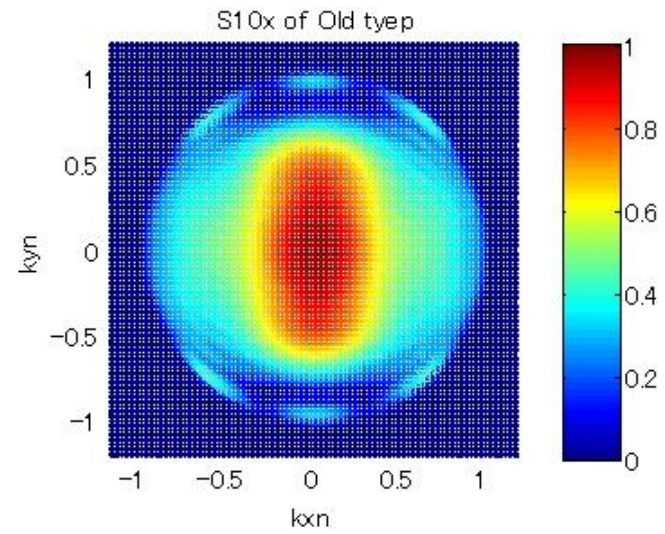

(a) $x$ component of $\mathbf{S}_{10}(\mathbf{K})$ (old type)

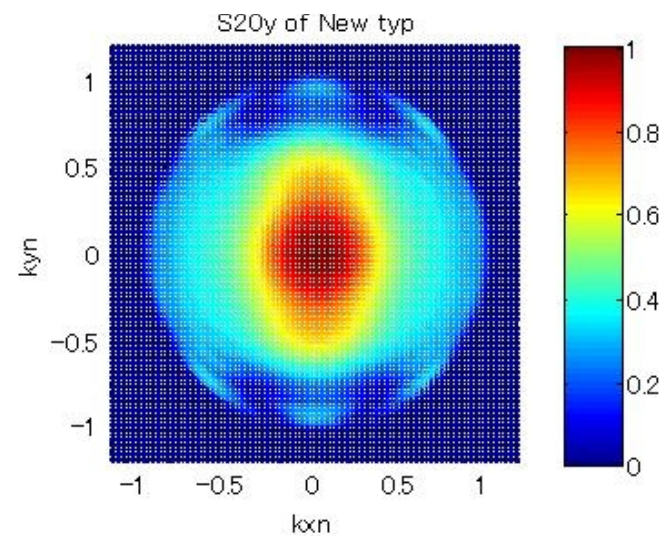

(b) $x$ component of $\mathbf{S}_{20}(\mathbf{K})$ (new type)

Fig. 10. $\mathbf{S}_{10}(\mathbf{K})$ (old type) and $\mathbf{S}_{20}(\mathbf{K})$ (new type) in $\mathbf{K}$ space whose magnitude is normalized by $k$ at 3.5 $\mathrm{GHz}$.

ferent from each other. This fact is true for other frequencies. Therefore the antenna gains of both antennas have different frequency characteristics as seen later. The phase variations of $\mathrm{F}\left(K_{n}\right)$ are slow and similar to the R-band standard horn antenna in Fig. 5. However the magnitude of $\mathrm{F}\left(K_{n}\right)$ decreases gradually compared to that in Fig. 5, since the pattern of the double-ridged waveguide horn is wider than the standard horn.

\section{4-3 New NGC and Gain}

The near-field gains of both types of antennas were measured at $z=1.5 \mathrm{~m}, 3 \mathrm{~m}$, and $3.87 \mathrm{~m}$, using the three antenna method (one in the old type, and two in the new type) [1] and compared with the ones obtained by using the new NGCs that were calculated through (15) to (17) using the measured data in Fig. 12.

Since the two antennas of new type had the same $\mathbf{S}_{20}(\mathbf{K})$ in this case, the $C_{22}(z)$ is calculated by (15) where the subscript 1 is replaced to 2 . Then the $\mathrm{NGC}_{2}(z)$ is equal to $C_{22}(z)$ as shown by (8). On the other hand, the $\operatorname{NGC}_{1}(z)$ is determined by (7) where the

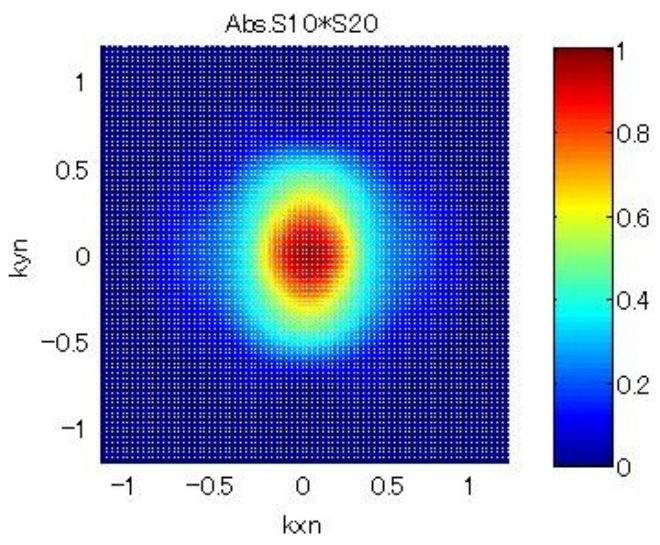

(a) Magnitude of $\mathbf{S}_{10}(-\mathbf{K}) \cdot \mathbf{S}_{20}(\mathbf{K})$

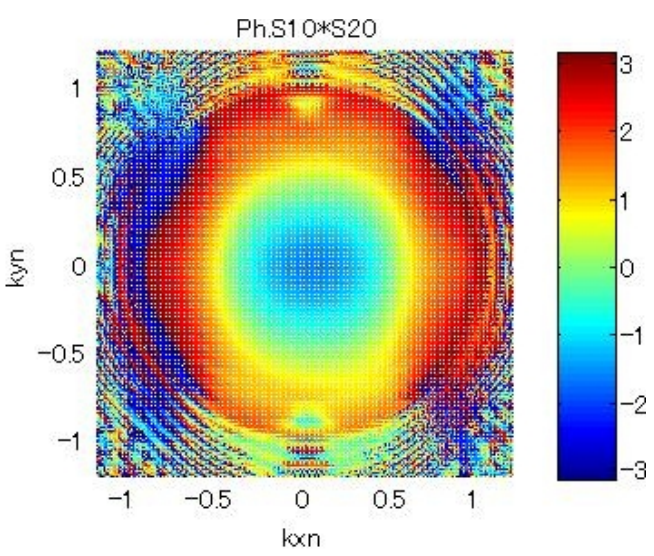

(b) Phase of $\mathbf{S}_{10}(-\mathbf{K}) \cdot \mathbf{S}_{20}(\mathbf{K})$

Fig. 11. Distribution of $\mathbf{S}_{10}(-\mathbf{K}) \cdot \mathbf{S}_{20}(\mathbf{K})$ in $\mathrm{K}$ space normalized to $k$ at $3.5 \mathrm{GHz}$.

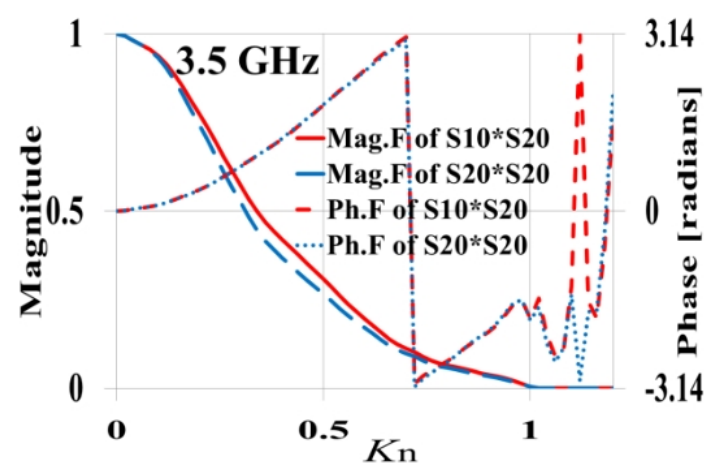

Fig. 12. Magnitude and phase of $\mathrm{F}\left(K_{n}\right)$ normalized to the value at $K_{n}=0$ at $3.5 \mathrm{GHz}$.

subscript 3 is replace to 2 as

$$
\mathrm{NGC}_{1}(z)=\frac{C_{12}^{2}(z)}{C_{22}(z)} .
$$

Fig. 13 compares the differences of the near-field gains relative to the near-field gains at $z=1.5 \mathrm{~m}$ and the $\mathrm{NGC}_{i}$ relative to the $\mathrm{NGC}_{i}$ at $z=1.5 \mathrm{~m}$. The differences obtained by both methods agree within $0.2 \mathrm{~dB}$ except 2 


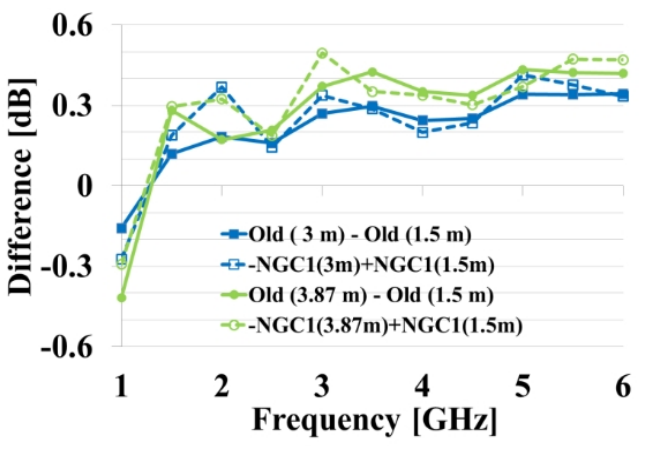

(a) Old type

0.9

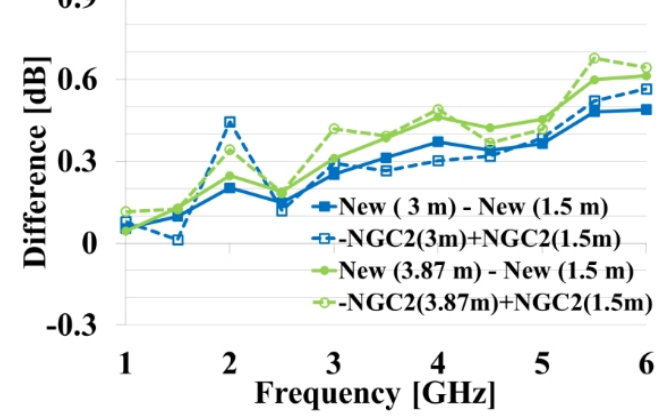

(b) New type

Fig. 13. Differences of the near-field gains by the three antenna method and those of the $\mathrm{NGCi}(i=1,2)$ by the new method. Each reference is the value at $z=1.5 \mathrm{~m}$.

$\mathrm{GHz}$ for the antenna of the new type. This is due to the large truncation error of $\mathbf{S}_{20}(\mathbf{K})$ that is not shown here. Overall, Fig. 13 demonstrates that the new method to calculate the NGC is effective even for broad beam-width antennas.

Finally we show the far-field gains obtained by the new NGC in Fig. 14. The far-field gain converted from the near-field gain at each $z$ is calculated by $\operatorname{NGC}_{1}(z)$ in (1) or $\mathrm{NGC}_{2}(z)\left(=C_{22}(z)\right)$ in (20). The far-field gain of the same antenna must be the same because $\operatorname{NGC}_{i}(z)$ converts the near-field gain at any $z$ to the same gain at infinity. Fig. 14 shows each gain agrees within 0.1 $\mathrm{dB}$ except $2 \mathrm{GHz}$ for both types and $1 \mathrm{GHz}$ for the old type. Therefore Fig. 14 demonstrates the usefulness of the new expression of the NGC combined with the planar near-field measurements using the photonic sensor, in order to measure the on-axis far-field gain and the on-axis near-field gain at any distance. In Fig. 14, the average far-field gain is calculated by averaging the far-field gains corrected by the corresponding NGCs from the near-field gains at $1.5 \mathrm{~m}, 3 \mathrm{~m}$, and $3.87 \mathrm{~m}$.

\section{Conclusion}

We have proposed a new expression of the near-field

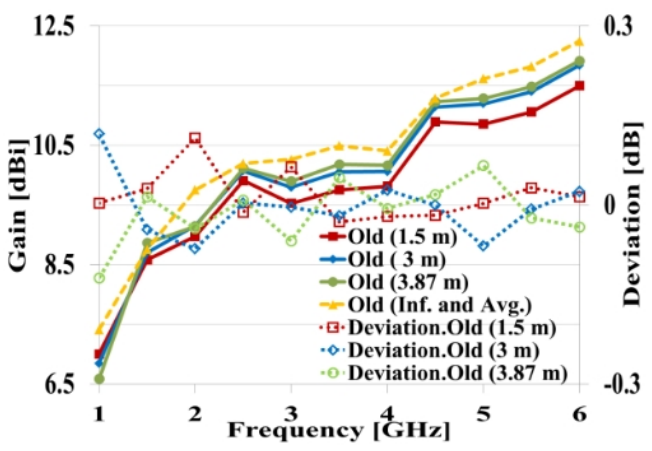

(a) Old type

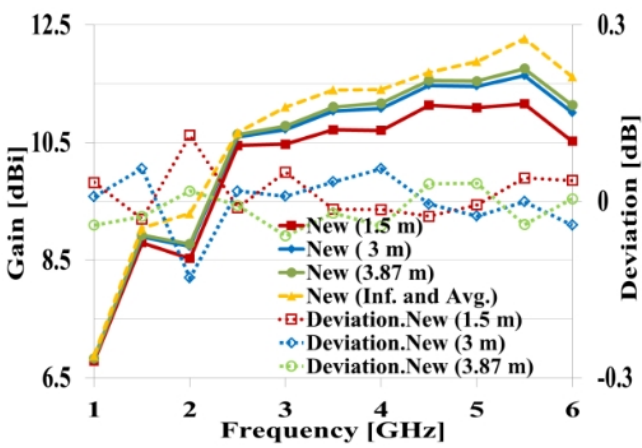

(b) New type

Fig. 14. Far-field gains (specified by Inf.) of antennas of the old and new types obtained by the new NGC at $z=1.5 \mathrm{~m}, 3 \mathrm{~m}, 3.87 \mathrm{~m}$. The deviation of each gain from the average far-field gain (Inf. and Avg.) is also shown.

gain correction to calculate the on-axis "far-field" gain from the on-axis "near-field" gain for a directive antenna.

The expression uses the transversal vectorial transmitting characteristic of the antenna that is closely related to the antenna pattern and is the Fourier transform of the radiated electric field on a plane measured by the planar near-field equipment.

The new expression has the advantages:

1. There is no need to correct $S_{21}$ measured by the VNA by using error terms representing the measurement system,

2. There is no need to measure the reflection coefficients of the antennas, and

3. The error terms representing the measurement system for each antenna measurement can be different, that is, the measurement condition can be changed for each antenna measurement.

Merits of using the photonic sensor as the probe are

4. It is easy to calculate the new NGC because the photonic sensor can detect directly the electric field on the plane,

5. The photonic sensor can measure the electric field 
without influence of unnecessary scattered fields because the sensor can be very close (a few centimeters) to the aperture of antennas.

We have validated the new NGC through the numerical simulation and demonstrated the usefulness of the new NGC in the measurements using the photonic sensor and the planar near-field equipment. The new expression realizes the measurements of the on-axis farfield gain for two kinds of double ridged waveguide horn antennas within $0.1 \mathrm{~dB}$ deviation at $1 \mathrm{GHz}$ to 6 GHz.

To improve the reliability of the measurements using the new NGC, we are now evaluating the uncertainty of the measurements, mainly, that of the photonic sensor system and will be submitted in the near future.

\section{References}

[1] G. E. Evans, Antenna Measurement Techniques, Artech House, pp. 19-22 and p. 66, 1990.

[2] T. S. Chu, R. A. Semplak, "Gain of electromagnetic horns," Bell System Technical Journal, vol. 44, no. 3, pp.527-537, Mar. 1965.

[3] A. C. Ludwig, R. A. Norman, "A new method for calculating correction factors for near-field gain measurements," IEEE Trans. Antennas and Propagation, vol. 21, no. 5, pp. 623-628, Sep. 1973.

[4] T. Uno, S. Adachi, "Range distance requirements for large antenna measurements," IEEE Trans. Antennasand Propagation, vol. 37, no. 6, pp. 707-720, Jun.

\section{Masanobu Hirose}

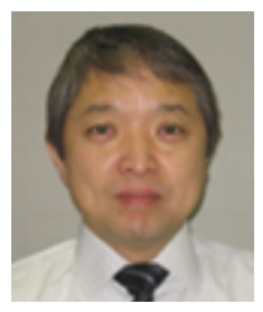

received the B.S. degree in Physics from Kanazawa University, Ishikawa, in 1979, andthe M.S. degree in Physics from Hiroshima University, Hiroshima, Japan, in 1981, and the M.E. and D.E. degrees in Electrical Engineering from The University of Electro-Communications, Tokyo, Japan, in 1983 and 1999 respectively. He has been working as a senior researcher in Metrology Institute of Japan, National Institute of Advanced Industrial Science and Technology, Ibaraki, Japan since 2000. His research interests include numerical analysis and antenna measurements. He is a member of the IEICE and IEEE.
1989.

[5] G. Mayhew-Ridgers, J. W. Odendaal, and J. Joubert, "Horn antenna analysis as applied to the evaluation of the gain-transfer method," IEEE Trans. Instrumentation and Measurement, vol. 49, no. 5, pp. 949-958, Oct. 2000.

[6] A. C. Newell, R. C. Baird, and P. F. Wacker, "Accurate measurement of antenna gain and polarization at reduced distances by an extrapolation tequnique," $I E$ EE Trans. Antennas and Propagation, vol. 21, no. 4, pp. 418-431, Jul. 1973.

[7] D. M. Kern, Plane-Wave Scattering-Matrix Theory of Antennas and Antenna-Antenna Interactions, National Bureau of Standards Monograph 162, Ch. 2, 1981.

[8] A. C. Newell, "Error analysis techniques for planar near-field measurements," IEEE Trans. Antennas and Propagation, vol. 36, no. 6, pp. 754-768, Jun. 1988.

[9] M. Hirose, T. Ishizone, and K. Komiyama, "Antenna pattern measurements using photonic sensor for planar near-field measurement at $\mathrm{X}$ band," IEICE Trans. Communions, vol. E87-B, no. 3, pp. 727-734, Mar. 2004.

[10] R. E. Collin, Antennas and Radiowave Propagation, McGraw-Hill, pp. 284-286 and pp. 303-304, 1985.

[11] M. Hirose, S. Kurokawa, "Far-field gain measurement using a new expression of near-field gain correction and photonic sensor," Proceedings of ISAP 2011, FrB2-1, SE10_1002, Oct. 2011.

[12] FEKO, http://www.feko.info
Satoru Kurokawa

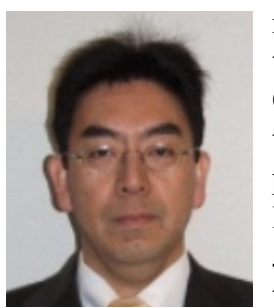

received B.E. and M.E. degrees in electrical engineering from Chiba University, Chiba, Japan, in 1987 and 1989, respectively, and a Ph.D. degree from the Department of Communication and Computer Engineering, Kyoto University, Kyoto, Japan, in 2003. He has been working as the electromagnetic field section chief with the Metrology Institute of Japan, National Institute of Advanced Industrial Science and Technology, Ibaraki, Japan, since 2003. His research interests include electromagnetic interference measurement, broadband antennas, time-domain measurements, microwave photonics. He is a member of the IEI$\mathrm{CE}$ and IEEE. 